\title{
A SURVEY ON THE STATE OF CLOUD ADOPTION IN MICRO \& SMALL BRAZILIAN IT COMPANIES
}

\author{
Gabriel Costa Silva ${ }^{1}$, Cesar Giovani Colini ${ }^{2}$ and Elizandro Ferreira ${ }^{2}$ \\ ${ }^{1}$ Universidade Tecnológica Federal do Paraná (UTFPR), Cornélio Procópio, Paraná, Brazil \\ ${ }^{2}$ Micro and Small Business Support Service (SEBRAE), Pato Branco, Paraná, Brazil
}

\begin{abstract}
Micro \& small companies are essential for Brazilian IT market. For instance, 95.5\% of Brazilian software development companies are micro or small. Although cloud computing plays a key role in reducing infrastructure and personnel costs in small companies, little is known on the state of cloud adoption in micro \& small Brazilian IT companies (MSBCs). If cloud adoption in MSBCs differs significantly from other companies around the world, MSBCs may need tailored training and tools. Moreover, these differences may limit MSBCs competitiveness in the global IT market. To understand the state of cloud adoption in MSBCs, we partnered with a regional IT association to conduct a survey with their 93 associated companies, from which we received 29 valid responses (31\% response rate). Eighty-three percent of surveyed companies are currently using cloud, $65 \%$ of cloud users in our survey use more than one cloud provider, and $54 \%$ of current cloud users reported issues when using cloud services. Compared to related surveys, we did not find significant differences regarding perceptions and practices of cloud adoption. This result suggests that cloud adoption may not vary significantly with company size and region.
\end{abstract}

\section{KEYWORDS}

Vale Digital, SEBRAE, Context-Driven Research, SMEs

\section{INTRODUCTION}

A recent survey shows that Brazilian domestic market of software and services reached the 9th position (US\$ 18.6 billion) in the 2017 world ranking, surpassing countries like Italy, Spain and India (ABES 2018). The same survey shows that $95.5 \%$ of Brazilian software development companies are micro (up to 10 empl.) or small (up to $100 \mathrm{empl}$.). As Richardson \& Von Wangenheim (2007) explain, small companies differ from medium and large in that they lack sufficient staff for specialised tasks and have tight budget. Therefore, cloud computing plays a key role in small companies since its unique characteristics may help reducing infrastructure and personnel costs (Marston et al. 2011).

However, little is known on the state of cloud adoption in micro \& small companies (Sahandi et al. 2013; Wood \& Buckley 2015). Furthermore, industrial cloud surveys show no data on perceptions and practices of Brazilian companies (RightScale 2018; Capgemini 2012; NorthBridge 2016). This lack of information impedes understanding the extent to which cloud adoption in micro \& small Brazilian IT companies (MSBCs) differs from other companies around the world. This understanding is essential for MSBCs since it: (i) enables benchmark against other companies; (ii) highlights regional differences; (iii) points out missing opportunities; and (iv) supports developing tailored tools.

Our research question asks whether perceptions and practices on cloud adoption in MSBCs differ significantly from other companies around the world. As sampling all MSBCs is an unrealistic goal because of the lack of a widely available list of all MSBCs (Baltes \& Diehl 2016), we undertook a context-driven survey. As Briand et al. (2017) explain, context-driven research "focuses on problems defined in collaboration with industrial partners and is driven by concrete needs in specific domains and development projects." Therefore, we partnered with a regional IT association (Vale Digital ${ }_{1}$ ) to supporting the region where our university lies in. This strategy enables us to contribute to the development of the region, firstly, by diagnosing the state of cloud adoption in regional companies, and secondly, by providing appropriate training, consultancy and tools.

\footnotetext{
${ }^{1}$ http://valedigital.org.br
} 
To address our research question, we conducted a survey research following rigorous guidelines (Section 2 ). We identified perceptions and practices of our respondents on cloud adoption, and issues faced when using cloud services (Section 3). When analysed against cloud surveys applied around the world, we observed that cloud adoption in MSBCs does not differ significantly from other companies although we could identify some interesting differences (Section 4). We present practical and research actions we are taking as our commitment with this context-driven survey, and explain the impact of our findings in our research question (Section 5). In Section 6, we discuss in detail several threats we identified. Finally, Section 7 concludes this paper.

\section{SURVEY \& PLAN EXECUTION}

Due to the lack of established guidelines for empirical and survey research in cloud computing (Gholami et al. 2016), our study is mainly based on Kitchenham \& Pfleeger (2008) guidelines as they enable rigorous survey preparation and execution. To encourage independent replications of this study, the questionnaires, the dataset, and the $\mathrm{R}$ instructions used to conduct and analyse this study are available online.

As Kitchenham \& Pfleeger (2008) recommend, we used previously published cloud surveys (NorthBridge 2016; RightScale 2018; Emison 2014; Capgemini 2012) to construct our questionnaires. These published surveys were applied worldwide, targeting different company sizes. We also used the only two surveys targeting Small and Medium Enterprises (SMEs) (Sahandi et al. 2013; Wood \& Buckley 2015) we could find.

From these surveys, we selected questions that could help answering our research question, and complemented our questionnaires with few questions we created to fully achieve our research goals. As recommended by Easterbrook et al. (2008), several questions included a short glossary to make clear to our respondents the meaning of terms we used in the survey, such as what we understand by service $\&$ deployment models. The research questionnaire consists of one open and 14 closed questions. Most closed questions present options from which our respondents could select one or more that apply in their companies. Cloud adoption questions target only companies that use or plan to use cloud services.

MSBCs are the target population of our survey. As most surveys (Kitchenham \& Pfleeger 2008; Baltes \& Diehl 2016), our survey uses a convenience sample since there is not a widely available list of all MSBCs. Unlike random sampling (probalistic), convenience sampling (non-probabilistic) obtains responses from participants that are available and willing to respond (Kitchenham \& Pfleeger 2008). As this study is undertaken as a context-driven research (Briand et al. 2017), our sample consists of companies that belong to a Brazilian IT association in the South-west of Paraná state, called Vale Digital 3 . The rationale for this choice is that Vale Digital lies in the same region where our university is located. Thus, we can contribute to the regional development by diagnosing the state of cloud adoption in regional companies and by providing training, consultancy and tools to support these companies.

Vale Digital groups 93 IT companies of varied sizes and ages. These companies are organised into four federations according to the macro-region where they lie in. We note that cloud surveys classify micro \& small companies in different ways (Sahandi et al. 2013; Wood \& Buckley 2015; RightScale 2018; ABES 2018). The Brazilian Micro and Small Business Support Service (SEBRAE 4 ) classifies a company size according to their number of employees. Whereas a micro company has up to 9 employees, a small company has up to 49 employees (SEBRAE \& DIEESE 2015). This classification is used as reference from hereon.

To get the list of associated companies, we directly approached each federation of Vale Digital by phone. During the phone call, we explained our purpose and main benefits of our study. The author of this study used his credibility in the region to asking for the list of associated companies, as recommended by Smith et al. (2013). After the phone call, we sent an email formalising our request. All four federations provided a full list of associated companies, containing their phone number, email and a person to contact.

As recommended by Kitchenham \& Pfleeger (2008), three researchers evaluated our questionnaires before we administered them. Suggestions given by researchers were analysed and the questionnaires were improved to the current version. To administer the questionnaires, we approached companies by phone to

\footnotetext{
${ }^{2}$ https://github.com/EmpiricalStudies/cloud-survey-valedigital.git

${ }^{3}$ http://valedigital.org.br

${ }^{4}$ https://www.sebrae.com.br/sites/PortalSebrae/canais_adicionais/sebrae_english
} 
identifying an appropriate respondent then, we sent the questionnaires to respondents by email. To increasing participation in our survey, we followed recommendations summarised by Smith et al. (2013). Thus, in the email to the respondent, we briefly explained the purpose of our survey, their benefits both to the respondent and to the region where the company lies in, and ensured the confidentiality of individual results.

\section{RESULTS}

Whereas this section identifies current perceptions and practices of MSBCs, the next section analyses our findings compared with that identified by related surveys in companies around the world. Together, these findings answer our research question within the context of our study.

\subsection{Company \& Respondent Characterisation}

From the ninety-three Vale Digital companies invited to our survey, forty responded to our online questionnaire (43\% of response rate). However, we removed 11 responses from our dataset because they came from companies larger than micro or small. Thus, our sample consists of twenty-nine valid responses ( $31 \%$ of response rate). It is important to highlight that even after the data reduction the sample size is still within the acceptable rate reported in the literature (Sahandi et al. 2013; Smith et al. 2013).

The responses came from nineteen micro (65.5\%) and ten small (34.5\%) companies. These companies employ from 1 to forty people. Twelve companies (41.3\%) have between 4 and 5 employees in total. Most companies (55.2\%) are older than 6 years, and more than one-third of companies in our sample are older than 10 years. Whereas only $10 \%$ of micro companies are older than 10 years, this percentage increases to $80 \%$ for small companies -- which may suggest a correlation between company age and size.

Thirteen companies $(44.8 \%)$ have software development as their main activity. Developing customisable software is the main activity of nearly one-third of our sample, followed by technical support, maintenance or other IT services (20.7\%). Surveyed companies target seventeen different markets. Technology and Consulting \& Services (55.2\% each) are the main target markets, followed by retail (48.3\%).

Owners, co-owners and CEOs quite often play technical roles in MSBCs. Not surprisingly, most (62\%) of our respondents are co-owners (41\%), CEOs (14\%) or owners (7\%). Only $38 \%$ of our respondents identified themselves as playing other roles in the company, namely: Director (24\%), Developer (7\%) and Manager (7\%). We note that these $38 \%$ of respondents employ in total $11 \%$ more employees than the other ones. This may suggest that larger companies assigned the questionnaire response to their employees whereas the owners responded the questionnaire in smaller companies.

Respondents have different levels of knowledge regarding cloud computing. In our characterisation questionnaire, basic knowledge (level 1, 24.1\%) and advanced knowledge (level 2, 27.6\%) do not imply practical experience managing cloud services. On the other hand, knowledge plus practical experience (level 3, 20.7\%), along with mastery (level 4, 27.6\%) do imply. We note that the sample size of respondents with and without practical experience is similar.

\subsection{Perceptions on Cloud Adoption}

The vast majority $(86.2 \%)$ of surveyed companies agree $(34.5 \%)$ or strongly agree $(51.7 \%)$ that using cloud computing leads to competitive advantages. This subset of companies is already cloud users or is planning to use cloud soon. Ten percent of companies that selected neutral to this question are already using cloud services. The only respondent that disagreed to this question (3.4\%) has no plan to use cloud computing.

Cost reduction (37.9\%), mobility \& convenience in accessing applications (27.6\%), and reduced capital expenditure in hardware, software, IT support or information security $(24.1 \%)$ are the three major drivers of cloud computing adoption, according to our respondents. Reduced planning, maximising resource utilisation, and increased computing capacity were cited only once.

Lack of knowledge, advice or guidelines on cloud adoption, along with fear of security breaches are major $(55.2 \%)$ barriers for cloud adoption in surveyed companies. Lack of trust, along with cost concerns are the second most cited concerns (31\%). Vendor lock-in was the less cited concern although this is a frequent issue faced by surveyed companies already using cloud (Section 3.3). 
Nearly half of our respondents (44\%) disagree or strongly disagree that there is sufficient advice, support $\&$ training for assisting MSBCs when selecting cloud technologies. On the other hand, only $10 \%$ agree or strongly agree with the existence of sufficient advice, support \& training for MSBCs. Forty-one percent of our respondents neither agreed nor disagreed with this question. We could not observe any significant demographics difference among respondents of this question.

Unauthorised access to or leak of information (51.7\%), service unavailability (48.3\%), and security breaches $(44.8 \%)$ are the three major risks of using cloud computing, according to our respondents. We observed a correlation between knowledge on cloud and perceptions on risks since only $32 \%$ of respondents who pointed security concerns as risks of using cloud have practical experience using cloud services.

Finally, several respondents who pointed service unavailability as a risk of using cloud computing noted on the survey constant issues with the internet service availability. This may suggest a major concern with the internet service unavailability rather than the cloud service.

\subsection{Practices on Cloud Adoption}

Eighty-three percent of surveyed companies are currently using cloud, $14 \%$ are planning to use, and only one company (3.4\%) has no plans of using cloud. Among companies that use or are planning to use cloud computing, $89 \%$ use or plan to use cloud computing for an existing project.

The only company that has no plans of using cloud is a micro sized, older than 10 years company with 4 employees that provides technical support, maintenance \& other IT services. This company disagrees that cloud can offer competitive advantage to its users, and the respondent pointed having advanced knowledge on cloud. As we noted in Section 3.1, this knowledge level does not imply practical cloud experience.

Not only the cloud adoption is high, but also the interest in providing cloud services. Sixteen companies (55.2\%) currently provide cloud services whereas $17.5 \%$ are planning to provide cloud services in the next 12 months. In addition, other $17.5 \%$ of companies are considering to provide cloud services in the future.

Only three companies $(10.3 \%)$ have no plans to provide cloud services. All these three companies are micro sized companies, but they differ in their main activities - software development (on request), technical support, maintenance \& other IT services, and other. Application servers is the main target for replacement with cloud service (28.6\%), followed by backup (21.4\%) and file storage (17.9\%).

Taking into consideration only companies that are currently using or are planning to use cloud computing, modern technology, along with competitive price are deemed as important criteria to select cloud providers for $60.7 \%$ of our respondents. Provider location and time in the market were the less cited criteria $(3.6 \%)$. We observed that two-thirds of companies $(\mathrm{n}=10)$ that pointed technical support as an important criterion for cloud provider selection have no practical experience with cloud service. This might suggest that they need technical support to compensate their lack of practical experience.

Google (41.7\%), Amazon (37.5\%), and Microsoft alongside IBM (12.5\% each) are the public cloud providers often used by cloud users in our survey. We noted that Google is preferred by companies using primarily SaaS services whereas Amazon is preferred by companies using primarily IaaS services.

Several cloud providers were cited only once (e.g., Heroku). In total, other providers are used by $54.2 \%$ of cloud users in our survey. We observed that $35 \%$ of cloud users in our survey use only one cloud provider whereas $65 \%$ of cloud users use more than one cloud provider. The practical knowledge on cloud computing is slightly greater $(64 \%)$ on those who use more than one cloud provider than on those who use only one (50\%), suggesting that practical experience may lead to confidence to use multiple providers.

Among companies that use or are planning to use cloud computing, $64 \%$ use or plan to use SaaS, followed by $54 \%$ for IaaS, and 29\% for PaaS. Other service models correspond to $19 \%$. Regarding the deployment model, companies using or planning to use cloud computing prefer the private model (39\%). Yet, public and hybrid models (each) are used or planned to be used by $29 \%$.

Fifty-four percent of current cloud users reported facing issues with cloud computing. The most often (always or usually happen) issues are vendor lock-in (37.5\%) and lack of qualified staff (25\%) whereas the less frequent (never or occasionally happen) are provider going out of business (100\%), unauthorised access (96\%) and loss of control (88\%). Only one company reported having no issue at all. This is a micro sized company established between 6 to 10 years, with some practical knowledge on cloud. This figure increases to 3 when we consider never or occasionally had any issue. 
We investigated the correlation between reported issues and the 3 major risks of using cloud computing (according to the perception of our respondents). Because only companies that are currently using cloud can inform the occurrence of issues, this analysis takes into account only companies that already use cloud.

We observed that 58\% of companies that consider unauthorised access as a risk of using cloud computing have never had any issue with unauthorised access. The figure increases to $92 \%$ if we consider companies that never or occasionally had any issue with unauthorised access. Fifty percent of companies that consider service unavailability as a risk have never or occasionally faced this issue. Similarly, $60 \%$ of companies that pointed security as a risk have never or occasionally faced this issue.

No company that reported unauthorised access, service unavailability or security breach as risks of using cloud computing faced these issues often (i.e., always or usually happen). This might suggest that the perception of cloud risks is likely to be based on assumptions, perhaps prompted by the lack of knowledge, rather than result of real negative experiences. On the other hand, we should consider that company's perception on risks might be prompted by the damage size rather than by the frequency of issues.

We also investigated the correlation between the 2 most often (i.e., always or usually happen) reported issues faced by cloud users and the barriers they pointed for cloud adoption. Again, we consider in this correlation only companies that are currently using cloud computing.

No respondent that often faced vendor lock-in issues mentioned it as a barrier for adoption. In contrast, $64 \%$ of companies that are facing issues with the lack of qualified staff pointed a barrier related to the lack of knowledge (that could be resolved by having qualified staff).

\section{ANALYSIS}

This section analyses the results of our survey (Section 3) compared with related surveys. This section analyses the set of surveys used as primary basis for structuring the questions used in this study (Section 2). We use the most relevant topics presented in Section 3 criteria for analysing this and related surveys.

\subsection{Drivers of Cloud Adoption}

Cost reduction is deemed the main driver for cloud adoption in three out of five surveys. Furthermore, cost is one of the three most cited drivers in Emison and NorthBridge's survey. These results may suggest that cloud adoption is cost-driven. Like Sahandi, Alkhalil \& Opara-Martins' survey, our respondents regard mobility and convenience in accessing applications as important drivers of cloud adoption. This may suggest that small companies have similar perceptions on cloud drivers, regardless of the country. On the other hand, scalability, which is among the most cited drivers in Emison, NorthBridge, and Sahandi, Alkhalil \& Opara-Martins' surveys, does not appear in our survey as one of the most cited drivers. In fact, increased computing capacity (which is one facet of scalability) is the less cited driver in our survey. This may suggest that our respondents differ from others in that they are primarily interested in reducing their costs.

\subsection{Cloud Adoption Barriers \& Support}

Like our survey, security concerns are among the most cited barriers for cloud adoption in related surveys. Similar to RightScale's survey, our respondents pointed out the lack of knowledge as an important barrier.

On the other hand, vendor lock-in is deemed as an important barrier for NorthBridge and Sahandi, Alkhalil \& Opara-Martins' surveys, but it is the less cited barrier in our survey. Another major difference between ours and related surveys is that several barriers tie in our survey.

For instance, two barriers share the position of most cited barrier, and three barriers share the 3rd position. This may suggest that our respondents do not share a unified perception on cloud adoption barriers. As in the drivers of cloud adoption, perhaps this result is correlated with different knowledge of our respondents on cloud (Section 3.1). In addition, the RightScale's survey shows that the level of importance on cloud barriers decreases with higher cloud maturity (RightScale 2018).

Only our survey asked about the support for cloud adoption. However, Wood \& Buckley (2015) note that most of their respondents "feel there is not enough advice, support or training available for SMEs," which is in line with our findings (Section 3.2). In addition, we observe that RightScale's survey points the lack of 
resources/expertise as one of the greatest barriers for cloud adoption - and this importance increases with lower cloud maturity (RightScale 2018). This might suggest that companies are not able to train their teams in the rate they need even when there is enough support for cloud adoption.

\subsection{Cloud Risks \& Adoption}

Security-related concerns are among the most cited risks of using cloud services. We observe that respondents in our and in related surveys share similar opinions. For instance, unauthorised access to or leak of information is among the three most cited risks in Emison's survey. Service unavailability, our 2nd most cited risk, appears as the 3rd most cited risk in the Wood \& Buckley's survey.

Apart from Sahandiet al. (2013) and Wood \& Buckley (2015) that do not investigate the rate of cloud adoption, the cloud adoption rate varies from $76 \%$ (Capgemini) to $96 \%$ (RightScale). We could not observe a correlation between the date when the survey was undertaken and cloud adoption rate, although the latest RightScale's survey shows an increase in the cloud adoption rate.

\subsection{Public Cloud Providers}

Among related surveys, only RightScale and Emison's survey investigated the most used public cloud providers. Like our survey, Amazon, Microsoft and Google are among the most used public cloud providers. However, the order and adoption rates vary. Whereas Google features as the most used provider in our survey (41.7\% of use), it features as the third most used in both RightScale and Emison's survey (18\% and $29 \%$, respectively). Microsoft, which features as the third most used provider in our survey ( $12.5 \%$ of use), features as the second most used in both RightScale and Emison's survey (45\% and 48\%, respectively). Finally, Amazon features as the second most used provider in our survey ( $37.5 \%$ of use) whereas it features as the most used provider in both RightScale and Emison's survey (64\% and 62\%, respectively).

RightScale's survey also shows the use rate of multiple cloud providers. Whereas $65 \%$ of our respondents are using more than one cloud provider, this figure decreases to $31 \%$ among RightScale's respondents. Taking into account only small \& medium companies investigated in RightScale's survey, the figure increases to $33 \%$ - suggesting that the number of cloud providers used inversely increases to company's size.

\section{DISCUSSION}

Several findings suggest that the lack of qualified staff may be a relevant issue faced by MSBCs when adopting cloud. Firstly, the lack of knowledge, advice or guidelines is pointed as the main barrier for cloud adoption. Secondly, a large number of respondents disagree that there is sufficient advice, support \& training for assisting their companies when adopting cloud technologies. Next, we observed a possible correlation between the level of knowledge on cloud and some perceptions and practices. Finally, we identified several differences between perceptions on risks and barriers when compared with real issues faced by cloud users.

As part of our commitment with this context-driven survey, we have been investigating cloud tools and platforms that could either facilitate the start of cloud beginners (Silva et al. 2018) or offer better performance (Ré et al. 2018). These studies offer valuable recommendations in the decision making on moving to/using the cloud. In addition, we are delivering cloud courses tailored to regional companies.

The research question driving our study asks whether perceptions and practices on cloud adoption in MSBCs differ significantly from other companies around the world. This investigation is critical for (i) enabling benchmark against other companies; (ii) highlighting regional differences; (iii) pointing out missing opportunities; and (iv) supporting the development of tailored tools.

Taking into account the results of our survey, when compared with companies in related surveys, MSBCs share similar perceptions on cloud drivers, barriers and risks. When compared only with SMEs, MSBCs face similar challenges, such as the lack of support for cloud adoption. MSBCs also share similar practices with companies in related surveys, such as wide SaaS adoption followed by IaaS, and their choice for cloud providers. Finally, cloud adoption rate in MSBCs is within the range identified in related surveys. These findings show that cloud adoption in MSBCs is in line with other companies around the world. Moreover, these findings suggest that cloud adoption may not vary significantly with company size and region. 
However, we found several differences that may have been prompted by regional influences, company size, cloud maturity, and primary sector. These differences need further analysis, which we plan to undertake in future work by: (i) replicating an extended version of this survey covering different company sizes and regions in the Paraná state, Brazil; (ii) interviewing respondents to identifying reasons that led to their decisions; and (iii) evaluating the impact of our training and research activities over regional companies.

\section{THREATS TO VALIDITY}

Internal threats impact the cause-effect relationship. These threats might lead to an alternative cause for the effect (Wohlin et al., 2012). We identified three internal threats. First, the instrument used to collect data (Section 2) could introduce some flaws due to different perceptions on cloud concepts, and due to its length. To reduce this threat, we provided a glossary in our questionnaires to explain main cloud concepts. In addition, we tested the questionnaires with a group of researchers before administering them to the sample population. In our tests, no respondent exceeded 15 minutes, which we considered as acceptable.

Next, our respondents' answers may not correspond to their real practice (Section 3.3). As data was collected online, we have no means to validate responses. However, we should point out that our respondents' answers are in line with worldwide surveys in several cases. A future replication of this survey should also consider in loco interviews for identifying possible deviations from reality.

Finally, different company sizes, regions, primary sectors and respondents sampled in related surveys make it difficult to carry out a fair analysis. As related surveys do not show categorised data, we were unable to make a fair comparison. This may have impacted our conclusion.

External threats restrict generalisation beyond the scope of the study (Wohlin et al. 2012). We identified three external threats. First, the timelessness of our survey results is a possible threat. Several indicators may change rapidly in cloud surveys, such as the rate of cloud adoption (Emison 2014). On the other hand, drivers and barriers do not tend to change significantly to switch positions (Emison 2014). However, results were presented to companies as soon as we consolidated them. In addition, we have been working on these results since we finished the survey. Therefore, expected results of the context-driven research have been met.

Second, one can argue whether our sample is representative of the target population. When surveying software developers, Baltes \& Diehl (2016) observe the lack of structured and systematic source of population representatives. Therefore, it is not uncommon to limit studies to a single group (Robillard 2009; Genero et al. 2003) - which is the case of our survey. To make the results of our survey valid to a group, we mitigated this threat by undertaking our survey as a context-driven research (Briand et al.2017). This enabled us to take full advantage of our survey results to contribute to regional MSBCs. In addition, our results can be regarded as a preliminary snapshot of the state of cloud adoption in MSBCs.

Finally, our sample may be considered small when compared with related surveys although our response rate is far above the minimum considered acceptable (Sahandi et al. 2013) and within the range often found in other surveys (Smith et al. 2013). Moreover, the literature emphasises that generalisation cannot be achieved by a single study, but independent replications are necessary to increase the confidence in empirical results and enable their generalisation beyond their initial context (Wohlin et al. 2012; Shull \& Feldmann 2008; Kitchenham et al. 2002). Therefore, we understand that our results are indicative and cannot be widely generalised beyond Vale Digital before replication and extension by other researchers.

\section{CONCLUSION}

The survey reported in this paper shows evidence that perceptions and practices on cloud adoption in micro \& small Brazilian IT companies (MSBCs) are in line with companies around the world. Although this suggests that cloud adoption does not vary significantly with the company size and region, we highlight that our findings are indicative and we do not claim for comprehensiveness or generalisation. Instead, by carrying out this survey as a context-driven research, we could devise tailored actions to helping regional MSBCs on addressing issues revealed by our survey. In addition, this study sheds some light to understand the state of cloud adoption in MSBCs, and its differences (or lack thereof) from other companies. 


\section{REFERENCES}

ABES 2018, Brazilian Software Market: scenario and trends, Technical report, Associação Brasileira das Empresas de Software (ABES). URL:https://goo.gl/DkUjMF

Baltes, S. \& Diehl, S. 2016, Worse Than Spam, in 'ESEM '16', ACM Press, New York, USA, pp. 1-6.

Briand, L., Bianculli, D., Nejati, S., Pastore, F. \& Sabetzadeh, M. (2017), 'The Case for Context-Driven Software Engineering Research: Generalizability Is Overrated', IEEE Software 34(5), 72-75.

Capgemini 2012, Business Cloud: The State of Play Shifts Rapidly, Technical report, Capgemini. URL:http://goo.gl/oES9Vn

Easterbrook, S., Singer, J., Storey, M.-A. \& Damian, D. 2008, Selecting Empirical Methods for Software Engineering Research, in 'Selecting Empirical Methods for SE Research', Springer London, chapter 11, pp. 285-311.

Emison, J. M. 2014, State of Cloud Survey, Technical report, InformationWeek. URL:http://goo.gl/GBx2NA

Genero, M., Piattini, M., Manso, E. \& Cantone, G. 2003, Building UML class diagram maintainability prediction models based on early metrics, in 'METRICS, 2003', IEEE Comput. Soc, Sydney, pp. 263-275.

Gholami, M. F., Daneshgar, F., Low, G. \& Beydoun, G. 2016, 'Cloud migration process - A survey, evaluation framework, and open challenges', Journal of Systems and Software 120, 31-69.

Kitchenham, B. A. \& Pfleeger, S. 2008, Personal Opinion Surveys, in F. Shull, J. Singer \& D. Sjøberg, eds, 'Guide to Advanced Empirical Software Engineering', Springer London, London, pp. 63-92.

Kitchenham, B. A., Pfleeger, S., Pickard, L., Jones, P., Hoaglin, D., El Emam, K. \& Rosenberg, J. 2002, 'Preliminary guidelines for empirical research in SE', IEEE TSE 28(8), 721-734.

Marston, S., Li, Z., Bandyopadhyay, S., Zhang, J. \& Ghalsasi, A. 2011, 'Cloud computing - The business perspective', Decision Support Systems 51(1), 176-189.

NorthBridge 2016, 2016 Future of Cloud Computing, Technical report, NorthBridge. URL:https://goo.gl/PnpjcR

Ré, R., Meloca, R. M., Roma, D. N., Ismael, M. A. d. C. \& Silva, G. C. 2018, 'An empirical study for evaluating the performance of multi-cloud APIs', Future Generation Computer Systems 79, 726- 738.

Richardson, I. \& Von Wangenheim, C. G. 2007, 'GuestEditors' Introduction: Why are Small Software Organizations Different?', IEEE Software 24(1), 18-22.

RightScale 2018, State of the Cloud Report, Technical report, Right Scale. URL:https://goo.gl/iq2Sh9

Robillard, M. P. 2009, 'What Makes APIs Hard to Learn?', IEEE Software 26(6), 27-34.

Sahandi, R., Alkhalil, A. \& Opara-Martins, J. (2013), 'Cloud Computing from SMES Perspective: A Survey-based Investigation', Journal of Information Technology Management XXIV (1), 1-12.

SEBRAE \& DIEESE 2015, Anuário do Trabalho na Micro e Pequena Empresa, Technical report, SEBRAE, São Paulo, SP. URL:http://goo.gl/jWw5tE

Shull, F. \& Feldmann, R. L. 2008, Building Theories from Multiple Evidence Sources, in 'Guide to Advanced Empirical Software Engineering', 1 ed, Springer London, London, chapter 13, pp. 337-364.

Silva, G. C., Ré, R. \& Silva, M. A. G. 2018, Evaluating efficiency, effectiveness and satisfaction of AWS and azure from the perspective of cloud beginners, in 'CASCON '18, ACM, Markham, Ontario, Canada, pp. 114- 125.

Smith, E., Loftin, R., Murphy-Hill, E., Bird, C. \& Zimmermann, T. (2013), Improving developer participation rates in surveys, in 'CHASE', IEEE, pp. 89-92.

Torchiano, M., Di Penta, M., Ricca, F., De Lucia, A. \&Lanubile, F. 2011, 'Migration of information systems in the Italian industry: A state of the practice survey', Information and Software Technology 53(1), 71-86.

Wohlin, C., Runeson, P., Höst, M., Ohlsson, M. C., Regnell, B. \& Wesslén, A. 2012, Experimentation in Software Engineering, Springer.

Wood, K. \& Buckley, K. (2015), Reality Vs Hype - Does Cloud Computing Meet the Expectations of SMEs?, in 'CLOSER '15', SCITEPRESS, Lisbon, Portugal, pp. 172-177. 